\title{
OUTFLOWING MOLECULAR GAS IN NGC 3079
}

\author{
J. A. IRWIN \\ Dept. of Physics, Queen's University, Kingston, Canada, K7L $3 N 6$
}

\author{
Y. SOFUE \\ Institute of Astronomy, The University of Tokyo, Mitaka, Tokyo 181
}

\begin{abstract}
NMA}^{12} \mathrm{CO}(\mathrm{J}=1-0)$ observations of the radio lobe spiral galaxy, NGC 3079, reveal a dense, rigidly rotating ring of molecular gas with a radius of about $750 \mathrm{pc}$. In addition, a tilted $\mathrm{CO}$ component as well as several molecular gas "spurs" are interpreted as linear features which are accelerated outwards by the nuclear outflow.
\end{abstract}

\section{INTRODUCTION AND OBSERVATIONS}

NGC 3079, an edge-on spiral galaxy, is noteworthy for its unusual kpc- scale radio lobes extending perpendicular to the plane (Duric et al. 1983) and apparently originating from a compact AGN (Irwin and Seaquist 1988). The recent discovery of a dense, nuclear molecular disk in this galaxy (Young et al. 1988) prompted this investigation to map the molecular gas distribution with the Nobeyama $\mathrm{Mm}$-wave Array (NMA).

CO $(\mathrm{J}=1-0)$ was mapped in the "C" and " $\mathrm{D}$ " configurations of the NMA, resulting in a HPBW of $4.1^{\prime \prime} \times 3.6^{\prime \prime}(308 \times 268 \mathrm{pc}$ at a distance of $15.6 \mathrm{Mpc})$. Sixteen frequency channels were averaged together, for a final spectral resolution of $13 \mathrm{~km} \mathrm{~s}^{-1}$, and the data were Fourier Transformed and "cleaned" in the usual way.

\section{RESULTS}

A nuclear molecular disk has been detected along the galaxy's major axis (PA= $-15^{\circ}$ ), containing a total $\mathrm{H}_{2}$ mass of $10.6 \times 10^{9}(\mathrm{D} / 15.6 \mathrm{Mpc})^{2} \mathrm{M}_{0}$. This is 50 - $60 \%$ of the total dynamical mass in the same region, as estimated from the rotation velocity, and is high compared to "normal" galaxies but appears to be typical of the central regions of $\mathrm{CO}$ or IR bright galaxies (e.g. Young and Scoville 1991). Within $400 \mathrm{pc}$, the average molecular hydrogen density is $\mathrm{n}\left(\mathrm{H}_{2}\right)$ $=530(\mathrm{D} / 15.6 \mathrm{Mpc})^{-1} \mathrm{~cm}^{-3}$, and the gas is in solid body rotation, achieving $\mathrm{V}_{\max }=330 \mathrm{~km} \mathrm{~s}^{-1}$. Flatter rotation occurs in the outer disk, and beyond $\approx$ $750 \mathrm{pc}$, the disk is distorted.

A "tilted component", inclined about $40^{\circ}$ from the major axis (i.e. $\mathrm{PA}=$ $\left.-55^{\circ}\right)$, is conspicuous in several channels around $V_{s y s}$. Spurs perpendicular to this tilted component are also visible, with the whole structure resembling an inclined "cross". Overlays of the radio continuum emission at various spatial resolutions 
show that the tilted component is aligned with the direction of the VLBI jet, and the perpendicular spurs occur in directions towards the outer radio lobes. The tilted component, itself, is present as a distinct velocity feature, remaining very close to $V_{\text {sys }}$ at all radii, whereas the velocity of the disk emission rises rapidly with radius, i.e. the tilted component does not appear to be rotating. Position - velocity cuts also show no evidence for expansion or contraction.

\section{DISCUSSION}

The velocity signature of the tilted component, its alignment with the VLBI jet direction, and the additional fact that it extends out beyond the NW and SE boundaries of the main disk suggest that this is a linear feature which is being ejected by the nuclear outflow. The perpendicular $\mathrm{CO}$ spurs towards the outer radio lobes, are similarly interpreted. This situation may be analogous to that in NGC 4258, in which the molecular clouds are recoiling due to the impacting of the "anomalous arms" (i.e. jets) (Plante et al. 1991). In NGC 3079, however, the apparent presence of two distinct, mutually perpendicular orientations near the nucleus, adds further complexity to the nuclear jet - ISM interaction in this galaxy. The orientations occur at $\mathrm{PA}=-56 /+124^{\circ}$ (VLBI jet direction, $\mathrm{CO}$ tilted component, and an inner radio continuum ridge), and at $\mathrm{PA}=$ $+34 /+214^{\circ}$ (brightest radio continuum ridges and $\mathrm{CO}$ spurs) - with features farther from the nucleus and/or galactic plane aligning more closely with the galaxy's minor axis. We speculate that a single bipolar jet may be precessing over a $90^{\circ}$ angle (in projection), or that a double or quadrupolar jet may be present. The latter possibility is reminiscent of the spiral galaxy, NGC 1097 (Wehrle et al. 1991, and references therein) in which two bipolar optical jets extend from the nucleus.

We refer the reader to the recently published papers by Sofue and Irwin (1992) and Irwin and Sofue (1992) for further information.

\section{REFERENCES}

Duric, N., Seaquist, E. R., and Davis, L. E. 1983, ApJ, 273, L11.

Irwin, J. A., and Seaquist, E. R. 1988, $A p J, 335,658$.

Irwin, J. A., and Sofue, Y. 1992, $A p J, 396, \mathrm{~L} 75$.

Plante, R. L., Lo, K. Y., Roy, J-R., Martin, P., and Noreau, L. 1991, ApJ, 381, 110.

Sofue, Y., and Irwin, J. 1992, PASJ, 44, 353.

Wehrle, A. E., Keel, W. C., and Jones, D. L. 1991, in The Interpretation of Modern Synthesis Observations of Spiral Galaxies, eds. N. Duric and P. C. Crane (P.A.S.P. Conf. Series 18), 251.

Young, J. S., Claussen, M. J., and Scoville, N. Z. 1988, ApJ, 324, 115.

Young J. S., and Scoville, N. Z. 1991, ARAEA, 29, 581. 